\title{
In vitro antibacterial activity of combinations of fosfomycin, minocycline and polymyxin $B$ on pan-drug-resistant Acinetobacter baumannii
}

\author{
YAJUN ZHANG ${ }^{1}$, FENGZHE CHEN $^{1}$, ENHUA SUN $^{2}$, RUIPING MA $^{1}$, CHUNMEI QU $^{1}$ and LIXIAN MA ${ }^{1}$ \\ Departments of ${ }^{1}$ Infectious Diseases and ${ }^{2}$ Laboratory Medicine, Qilu Hospital, \\ Shandong University, Jinan, Shandong 250012, P.R. China
}

Received December 16, 2012; Accepted March 22, 2013

DOI: $10.3892 /$ etm.2013.1039

\begin{abstract}
The aim of this study was to determine the effects of combinations of fosfomycin, minocycline and polymyxin B in the treatment of pan-drug-resistant Acinetobacter baumannii (PDR-Ab). The in vitro antibacterial activities of the drugs were evaluated by determination of the minimum inhibitory concentration (MIC) and the fractional inhibitory concentration index (FICI). A total of 25 strains of PDR-Ab were selected using the VITEK32 microbial analysis instrument and the Kirby-Bauer (K-B) method. A broth microdilution method was used to determine the MIC for each of the three drugs, and the checkerboard method was simultaneously used to determine the MICs for combinations of the drugs. FICI values were also calculated. While fosfomycin alone was ineffective for the treatment of PDR-Ab, its MIC value was significantly reduced when used in combination with minocycline or polymyxin B. The combined use of minocycline and polymyxin B also significantly reduced the MIC value of each drug. The FICI values revealed that the drugs had synergistic or additive effects when used in combination. The determination of the MIC and FICI values for the combinations of drugs demonstrated that there is synergistic or additive effect upon the combined use of fosfomycin with minocycline or polymyxin B. The combined use of minocycline and polymyxin $\mathrm{B}$ also results in a significant reduction in the MIC values of the two drugs. These experimental results may provide a basis for the future clinical treatment of Acinetobacter baumannii.
\end{abstract}

Correspondence to: Professor Lixian Ma, Department of Infectious Diseases, Qilu Hospital, Shandong University, 107 Wenhua West Road, Jinan, Shandong 250012, P.R. China E-mail: malx123456@163.com

Key words: pan-drug resistant Acinetobacter baumannii, minocycline, fosfomycin, polymyxin $\mathrm{B}$, minimum inhibitory concentration, fractional inhibitory concentration index

\section{Introduction}

Over the past decade, the emergence of multi-and even pan-drug-resistant Acinetobacter baumannii (PDR-Ab) has brought a tremendous challenge to hospital infection control and clinical treatment. As an opportunistic pathogen widely distributed in the hospital and the natural environment, Acinetobacter baumannii is normally colonized in the respiratory, digestive, urinary and reproductive systems. It causes nosocomial infections such as sepsis, ventilator-associated pneumonia and urinary tract infections under certain conditions (1). In recent years, the extensive use and misuse of antibiotics have led to the increasing resistance of Acinetobacter baumannii, which has attracted significant attention from relevant personnel.

Drugs that are potentially effective for the treatment of multi-resistant Acinetobacter baumannii include carbapenems, tetracyclines and enzyme inhibitors, and polymyxins (1). Polymyxins are regarded as the last line of defense. However, a single medication is typically ineffective in clinical treatment (1). Therefore, while it remains necessary to develop new drugs, at present, the only method for the treatment of Acinetobacter baumannii infection is the novel utilization of traditional drugs or the combined use of multiple drugs (2).

In the current study, we examined the effects of combinations of fosfomycin (3-5), minocycline (6) and polymyxin B (7) in the treatment of PDR-Ab. The three drugs were chosen as they show considerable effectiveness and are commonly used in combination in the treatment of multi-resistant bacteria. Our results suggest that the effects of multiple drugs used in combination are synergistic and additive, particularly for the combined use of polymyxin B and minocycline.

\section{Materials and methods}

Experimental strains. A total of 25 strains of PDR-Ab were collected from the Qilu Hospital of Shandong University (Jinan, China). They were identified to be resistant to multiple drugs, including carbapenems, quinolones, cephalosporins, aminoglycosides and sulfonamides, by the Kirby-Bauer (K-B) method. Among them, 24 strains were from sputum specimens 
Table I. Antimicrobial $\mathrm{MIC}_{\mathrm{G}}, \mathrm{MIC}_{50}$, and $\mathrm{MIC}_{90}$ values of fosfomycin, minocycline and polymyxin B $(\mu \mathrm{g} / \mathrm{ml})$.

\begin{tabular}{|c|c|c|c|c|}
\hline Antimicrobial drugs & $\mathrm{MIC}_{\text {range }}$ & $\mathrm{MIC}_{\mathrm{G}}$ & $\mathrm{MIC}_{50}$ & $\mathrm{MIC}_{90}$ \\
\hline Polymyxin B & $1-8$ & 3.8906 & 4 & 8 \\
\hline Minocycline & $4-16$ & 11.4716 & 16 & 16 \\
\hline Fosfomycin & $>512$ & $>512$ & - & - \\
\hline
\end{tabular}

$\mathrm{MIC}_{\text {range }}$, the range of the MIC values; $\mathrm{MIC}_{\mathrm{G}}$, the mean MIC value; $\mathrm{MIC}_{50}$, the MIC value at which $50 \%$ of bacteria were inhibited; $\mathrm{MIC}$, MIC value at which $90 \%$ of bacteria were inhibited.

Table II. MIC values of fosfomycin, minocycline and polymyxin B in combined use $(\mu \mathrm{g} / \mathrm{ml})$.

\begin{tabular}{|c|c|c|c|c|}
\hline Combined medication & $\mathrm{MIC}_{\text {range }}$ & $\mathrm{MIC}_{\mathrm{G}}$ & $\mathrm{MIC}_{50}$ & $\mathrm{MIC}_{90}$ \\
\hline Polymyxin B and & $0.25-4$ & 1.0281 & 1 & 2 \\
\hline minocycline & $0.25-8$ & 2.2974 & 2 & 8 \\
\hline Polymyxin B and & $0.25-4$ & 1.3195 & 2 & 4 \\
\hline fosfomycin & $64-1024$ & 484.381 & 512 & 1024 \\
\hline Minocycline and & $0.5-4$ & 2.1140 & 2 & 4 \\
\hline fosfomycin & $64-1024$ & 526.39 & 512 & 1024 \\
\hline
\end{tabular}

$\mathrm{MIC}_{\text {range }}$, the range of the MIC values; $\mathrm{MIC}_{\mathrm{G}}$, the mean MIC value; $\mathrm{MIC}_{50}$, the MIC value at which $50 \%$ of bacteria were inhibited; $\mathrm{MIC}$, the MIC value at which $90 \%$ of bacteria were inhibited. The MIC values of the three drugs when used alone were significantly different from those when used in combination $(\mathrm{P}<0.05)$.

and one was from wound secretions. The strains were stored at $-80^{\circ} \mathrm{C}$. Escherichia coli ATCC25922 was used as a quality control strain.

Materials and instruments. Minocycline and fosfomycin were purchased from the National Institute for the Control of Pharmaceutical and Biological Products (Beijing, China). Polymyxin B in this study was INALCO1758-9325 (Baierdi Biotechnology Company, Beijing, China). $\mathrm{MH}$ agar and $\mathrm{MH}$ broth were purchased from Boshang Biotechnology Company (Jinan, China). The VITEK32 microbial analysis instrument was purchased from Boshang Biotechnology Company.

Broth microdilution method. Bacterial suspensions were prepared by inoculation of colonies from a freshly cultured plate, followed by culturing for $4-6 \mathrm{~h}$ at $35^{\circ} \mathrm{C}$. The turbidity of the cultures was then calibrated to 0.5 McFarland $\left(1.5 \times 10^{8} \mathrm{CFU} / \mathrm{ml}\right)$ using a spectrophotometer. Stock solutions of antibiotics were prepared and stored at $-60^{\circ} \mathrm{C}$.

For the determination of the minimum inhibitory concentration (MIC) value of each drug, various concentrations of the drugs were added to a 96-well plate. Bacterial suspensions were added to each well at a final concentration of $1.5 \times 10^{5} \mathrm{CFU} / \mathrm{ml}$ and incubated at $35 \pm 2^{\circ} \mathrm{C}$ for $18-24 \mathrm{~h}$. The MIC value was determined as the drug concentration at which bacterial growth was completely inhibited.

For the joint drug susceptibility test, the fold drug dilutions were determined according to the MIC values of each drug. Each combination of two drugs at various concentrations was mixed with a bacterial suspension at a final concentration of $1.5 \times 10^{5} \mathrm{CFU} / \mathrm{ml}$, and incubated at $35 \pm 2^{\circ} \mathrm{C}$ for $18-24 \mathrm{~h}$. The
MIC values of each drug were recorded. The fractional inhibitory concentration index (FICI) was calculated as follows: $\mathrm{FICI}=\mathrm{MIC}_{\mathrm{A} 2} / \mathrm{MIC}_{\mathrm{A} 1}+\mathrm{MIC}_{\mathrm{B} 2} / \mathrm{MIC}_{\mathrm{B} 1}$, where $\mathrm{MIC}_{\mathrm{A} 2}$, the $\mathrm{MIC}$ value of drug $\mathrm{A}$ in combinative use; $\mathrm{MIC}_{\mathrm{A} 1}$, the $\mathrm{MIC}$ value of drug A used alone; $\mathrm{MIC}_{\mathrm{B} 2}$, the MIC value of drug B in combinative use; and $\mathrm{MIC}_{\mathrm{B} 1}$, the $\mathrm{MIC}$ value of drug $\mathrm{B}$ used alone. FICI values of $\leq 0.5,0.5-1.0,1.0-4.0$ and $>4.0$ were considered to indicate a synergistic effect, additive effect, independent effect and antagonistic effect, respectively (8).

Statistical analysis. SPSS 17.0 software (SPSS, Inc., Chicago, IL, USA) was used to perform the statistical analysis, using a paired t-test and the geometric mean. $\mathrm{P}<0.05$ was considered to indicate a statistically significant result.

\section{Results}

MIC values are significantly reduced when fosfomycin is used in combination with minocycline or polymyxin B. As shown in Tables I and II, the MIC values of fosfomycin, minocycline and polymyxin B when used in combination were significantly reduced when compared with those when used alone. Notably, while fosfomycin alone showed no significant antibacterial effects on Acinetobacter baumannii, its MIC value was significantly reduced when used in combination with minocycline or polymyxin B (Tables I and II). We further analyzed antibacterial effects for combinations of minocycline and polymyxin B. The results in Fig. 1 indicate that the MIC values of minocycline and polymyxin B when used in combination were significantly reduced when compared with those when used alone (Fig. 1). 
Table III. Fractional inhibitory concentration index (FICI) values of fosfomycin, minocycline and polymyxin B upon combined use

\begin{tabular}{|c|c|c|c|c|}
\hline Combined medication & FICI $(\leq 0.5)$ & FICI $(0.5-1)$ & FICI (1-4) & FICI $(>4)$ \\
\hline Polymyxin B and minocycline & $44 \%$ & $48 \%$ & $8 \%$ & 0 \\
\hline Polymyxin B and fosfomycin & $16 \%$ & $44 \%$ & $40 \%$ & 0 \\
\hline Minocycline and fosfomycin & $12 \%$ & $56 \%$ & $32 \%$ & 0 \\
\hline
\end{tabular}

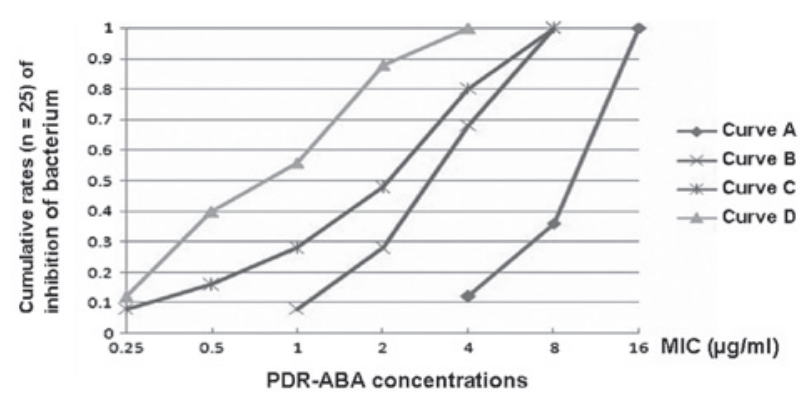

Figure 1. Antibacterial effects of minocycline, polymyxin B and their combinations. Curve A, concentrations of minocycline when used alone; curve B, concentrations of polymyxin $\mathrm{B}$ when used alone; curve $\mathrm{C}$, concentrations of minocycline when used in combination with polymyxin $\mathrm{B}$; curve $\mathrm{D}$, concentrations of polymyxin B when used in combination with minocycline. PDR-ABA, pan-drug-resistant Acinetobacter baumannii; MIC, minimum inhibitory concentration.

Effects of combinations of drugs. As demonstrated in Table III, the FICI values for combinations of minocycline and polymyxin B were generally $\leq 0.5$ or $>0.5-1.0$, suggesting that the two drugs have a synergistic or additive effect. However, the FICI values for fosfomycin and polymyxin B were mostly within the ranges $0.5-1.0$ and 1.0-4.0, suggesting that the effects of fosfomycin and polymyxin B were additive or independent. Similar results were also observed for fosfomycin and minocycline. No antagonistic effects for the drug combinations were observed. Based on these findings, the drugs when used in combination have synergistic, additive or independent effects, rather than antagonistic effects. The synergistic and additive effects were the most prominent, particularly for the combination of minocycline and polymyxin B.

\section{Discussion}

In the treatment of infections caused by pan-drug-resistant bacteria, a single medication is typically ineffective. Therefore, the combined use of multiple drugs is recommended. The medication regimen is usually designed by referring to the joint drug susceptibility test in vitro. For the evaluation of the combined effect of antimicrobial agents, the FICI value is an important parameter (9). It is considered that the combined use of drugs with a synergistic or additive effect as determined by the FICI is likely to be effective in clinical treatments.

In the current study, we examined the effects of combinations of fosfomycin, minocycline and polymyxin $\mathrm{B}$ in the treatment of PDR-Ab. Our results revealed that the MIC values of the drugs were reduced when they were used in combination, suggesting a synergistic or additive effect. This effect was further demonstrated by determining the FICI values.

Based on these findings, the combined use of multiple drugs is effective, which provides a basis for the use of drug combinations in the clinical treatment of PDR-Ab. Furthermore, it also has other advantages over the traditional use of a single medication. By using combinations of multiple drugs, bacterial resistance is likely to be reduced. The incidence of adverse drug responses is also likely to be reduced due to the use of lower drug doses.

\section{Acknowledgments}

This study was supported by the 2012 Innovation Fund (Free Exploration Grant type I, grant no. 26010172611152) of Shandong University.

\section{References}

1. Shi Y and Liu DW: Exploration of treatment of multi-drug resistant Acinetobacter. Prescription Drugs 9: 11-16, 2006 (In Chinese).

2. Liang W, Liu XF, Huang J, Zhu DM, Li J and Zhang J: Activities of colistin-and minocycline-based combinations against extensive drug resistant Acinetobacter baumannii isolates from intensive care unit patients. BMC Infect Dis 11: 109, 2011.

3. Lian JJ and Tang QY: Synergy and the clinical applications of fosfomycin as well as other antimicrobial agents. Foreign Medicine (Antibiotics) 1: 44-50, 2003 (In Chinese).

4. Pang XJ, Chen Y and Lu MD: Time difference attack therapy producing ESBLs G - 22 cases of bacillus infection. Chinese Journal of New Drugs 3: 351-352, 2005 (In Chinese).

5. Pang XJ, Zhou HW and Wei H: Clinical studies of the treatment of Pan-drug resistant Acinetobacter baumannii infection. Chinese Journal of Pharmaceutical Sciences 5: 397-398, 2009 (In Chinese).

6. Huang ZG, Huang C and Ma DY: Antibacterial activity of minocycline on Acinetobacter baumannii. International Journal of Laboratory Medicine 10: 1155-1157, 2010 (In Chinese).

7. Shi YB and Dong Y: In vitro studies of tigecycline and polymyxin B treatments on pan-drug resistant Acinetobacter baumannii strains resistant to cefoperazone. Zhongguo weishengtaixue zazhi 9: 825-827, 2010 (In Chinese).

8. Sopirala MM, Mangino JE, Gebreyes WA, et al: Synergy testing by Etest, microdilution checkerboard, and time-kill methods for pan-drug-resistant Acinetobacter baumannii. Antimicrob Agents Chemother 54: 4678-4683, 2010.

9. Lee YS, Kang OH, Choi JG, et al: Synergistic effect of emodin in combination with ampicillin or oxacillin against methicillin-resistant Staphylococcus aureus. Pharm Biol 48: 1285-1290, 2010 